Revue d'histoire de l'Amérique française

ALS REVUE D.HISTOIRE DE L'AMÉRIQUE FRANÇAISE

\title{
Reconstitution de la population du Canada au 30 juin 1663 suivant Marcel Trudel
}

\section{Hubert Charbonneau}

Volume 27, numéro 3, décembre 1973

URI : https://id.erudit.org/iderudit/303286ar

DOI : https://doi.org/10.7202/303286ar

Aller au sommaire du numéro

\section{Éditeur(s)}

Institut d'histoire de l'Amérique française

\section{ISSN}

0035-2357 (imprimé)

1492-1383 (numérique)

Découvrir la revue

\section{Citer cette note}

Charbonneau, H. (1973). Reconstitution de la population du Canada au 30 juin 1663 suivant Marcel Trudel. Revue d'histoire de l'Amérique française, 27(3),

417-424. https://doi.org/10.7202/303286ar d'utilisation que vous pouvez consulter en ligne. 


\section{RECONSTITUTION DE LA POPULATION DU CANADA AU 30 JUIN 1663 SUIVANT MARCEL TRUDEL ${ }^{1}$}

Sans données statistiques, il n'y a pas de démographie possible. Comme la création des organismes de statistique ne remonte guère au-delà de 1800 , les sources chiffrées font le plus souvent défaut pour les périodes antérieures au $\mathrm{XIX}^{\mathrm{e}}$ siècle. Aussi, nos connaissances sur les populations passées se révélaient-elles extrêmement médiocres jusqu'à une époque toute récente. Fort heureusement, les temps ont changé et la démographie historique connaît une expansion remarquable, depuis que démographes et historiens ont découvert les inépuisables ressources offertes par les archives. La reconstitution des faits de population à partir des vieux papiers demeure certes une entreprise ardue mais d'une exceptionnelle fécondité. Nos vues sur le passé s'en trouvent peu à peu changées et la science démographique elle-même en ressort revigorée.

Travailleur infatigable et d'une grande productivité, Marcel Trudel nous livrait récemment les résultats de recherches qui illustrent parfaitement la nature de ce nouveau champ d'action. $\mathrm{Au}$ prix de laborieuses compilations, il a su exploiter de nombreuses sources manuscrites et imprimées pour réaliser un véritable recensement de la population canadienne au 30 juin 1663 , c'est-à-dire à la fin de l'ère des Compagnies. La tentative était audacieuse mais réaliste, comme le laissaient présager la masse des archives et l'importance des travaux généalogiques.

L'ouvrage se divise en deux parties distinctes: le recensement reproduit en appendice et six chapitres de description analytique. Le relevé de la population porte sur 3,035 individus, classés principalement suivant l'ordre alphabétique des noms de famille dans chacun des trois gouvernements de Québec, Trois-Rivières et Montréal. Outre les caractéristiques nominatives, on dispose éventuellement pour chaque personne, de renseignements se rapportant aux rubriques suivantes: sexe, état matrimonial, âge, signature, profession, origine, "venu sous contrat", arpents en seigneurie, arpents en censive, emplacement en toises. Les gens d'Eglise sont traités à part. Dérivant naturel-

1 Marcel Trudel, La population du Canada en 166.3 (Montréal, les Editions Fides, 1973), XL-368 p., $23.5 \mathrm{~cm}$. Ill., cartes. Collection "Fleur de lys", études historiques canadiennes. 
lement de la collecte des données, les six chapitres portent les titres qui suivent: le nombre, l'origine des immigrants, l'âge et le sexe, l'état matrimonial, les professions, les groupes sociaux.

Accompagné d'un précieux index alphabétique général permettant de retrouver facilement les diverses personnes recensées, l'appendice s'étend sur 164 pages. Clairement présenté, il forme un nouvel et remarquable outil de travail pour tous ceux qui s'intéressent au $\mathrm{XVII}^{\mathrm{e}}$ siècle canadien. De lecture facile, les six chapitres sont écrits dans un style agréable en dépit de l'aridité du sujet. Dans l'ensemble, l'auteur s'en tient à la mesure des phénomènes, sans pousser l'analyse. Il faut dire que l'analyse démographique est forcément limitée quand on recourt aux données d'un seul recensement. Il est d'ailleurs remarquable que les commentaires les plus élaborés de l'ouvrage soient ceux qui empruntent aux diverses données de mouvement dont on a tiré l'état de la population de 1663. Sans doute, Marcel Trudel nous réserve-t-il ses vues d'historien dans son prochain ouvrage sur la période des Cent-Associés.

Contrairement à de trop nombreuses publications, l'illustration abonde, au bénéfice de la clarté et de la compréhension. Il arrive même qu'elle multiplie les recoupements (figures 7 et 8 par exemple), mais on ne s'en plaindra pas. Il faut toutefois relever quelques erreurs classiques qui entachent quelque peu ce travail et qu'on aurait pu facilement éviter. L'auteur tombe en effet dans le piège bien connu de l'histogramme: les effectifs des moins de 15 ans se révèlent surestimés dans les figures 19, 20 et 28. Rappelons à cet égard que les surfaces doivent être proportionnelles aux nombres qu'elles représentent. D'autre part c'est fausser la réalité que de répartir les "non déclaré" au bas de la pyramide des âges, comme le montrent les figures 15 et 16 : il aurait sûrement mieux valu les distribuer autrement, même de façon approximative. Enfin, certaines pyramides (figure 6 par exemple) ne sont pas conformes aux règles graphiques généralement admises: la hauteur doit être le plus possible égale aux deux tiers de la largeur totale environ.

Le premier chapitre est soigné. On y relève des développements intéressants sur les premiers Canadiens de naissance et sur le métissage. A la page 26, on peut se demander toutefois si c'est "la force des choses" ou plutôt l'absence d'une immigration vraiment organisée qui crée le fort rapport de masculinité chez les immigrants.

Au chapitre deux, les taux d'augmentation, dont il est fait état à la page 49 , pourraient se révéler trompeurs si on en 
dégageait des conclusions sur la natalité. Ces taux dépendent surtout de la durée d'implantation et on sait que les gens du Perche sont arrivés en moyenne assez tôt au Canada. Aussi, un tableau classant les immigrants suivant la durée d'implantation (première mention au Canada) et la province d'origine serait-il du plus grand intérêt. Quand M. Trudel met en relief le mouvement, réparti sur trois décennies, des 140 émigrants du Perche, il convient de rapporter ce nombre à la population d'origine. Même s'il s'agit d'une petite province d'environ 70,000 habitants, on ne saurait en aucun cas parler de "départs massifs" (page $38)^{2}$. A l'échelle de la France, l'émigration vers le Canada durant le XVII ${ }^{e}$ siècle, c'est la goutte d'eau dans l'océan. Il faudrait néanmoins tenter de dresser une carte détaillée des origines géographiques des immigrants en provenance des provinces les plus pourvoyeuses.

A la lecture du chapitre trois, il faudra se rappeler (page 58 notamment) qu'une distinction doit être clairement établie entre l'âge des immigrants et celui des immigrés: en 1663, certaines personnes sont au Canada depuis plus de 25 ans.

$\mathrm{Au}$ quatrième chapitre, l'étude des caractéristiques des couples retient l'attention, en particulier dans la comparaison des origines ou des signatures des conjoints. Mais le lecteur ne doit jamais oublier qu'il s'agit d'une situation à un moment précis. Quand Marcel Trudel nous dit à la fin de ce chapitre, qu'Hélène Desportes est "la plus féconde", il entend que c'est la femme qui a eu le plus d'enfants connus avant le 30 juin 1663. $\mathrm{Si}$ le déséquilibre sur le marché matrimonial est bien décrit, l'impression subsiste que toutes les jeunes filles devaient être nubiles à 12 ans, suivant les règlements de l'Eglise. En réalité, nos ancêtres étaient apparemment beaucoup moins précoces que leurs descendants $d u \mathrm{XX}^{\mathrm{e}}$ siècle et certaines jeunes filles ne devenaient alors nubiles que longtemps après l'âge minimal réglementaire. A la page 71, on est par ailleurs surpris de lire qu'une charge d'enfants était un obstacle au remariage: certaines études sur le XVIII ${ }^{e}$ siècle canadien n'ont pas abouti à cette conclusion ${ }^{3}$. L'enfant était une valeur économique appréciable à l'époque. Dans l'étude des lieux d'origine respectifs des

2 Voir à ce propos, Hubert Charbonneau, Tourouvre-au-Perche aux $X V I I^{e}$ et XVIIIe siècles, étude de démographie historique (Paris, Presses universitaires de France, 1970), XIV-423 p., $24 \mathrm{~cm}$. Ill., cartes. Cahier no 55 des "Travaux et documents de l'Institut national d'études démographiques".

${ }^{3}$ Louis Duchesne, Nuptialité et fécondabilité des veuves remariées: les Canadiennes au XVIII ${ }^{e}$ siècle. Mémoire de maîtrise soumis au Département de démographie de l'Université de Montréal (1972). 
conjoints, il conviendrait enfin de bien distinguer les couples mariés en France de ceux mariés au Canada, car il y eût sûrement plus de mélange dans le pays d'arrivée que dans celui de départ (page 81).

Les chapitres cinq et six donnent une excellente vue d'ensemble sur la société pionnière canadienne, quoique la place tenue par les cas non déterminés ne soit pas toujours aussi nettement établie que dans les chapitres précédents. Dans le classement des individus suivant la profession, on peut se demander s'il y a lieu de n'envisager qu'un seul métier. Il y avait sûrement des individus polyvalents, comme il y en avait d'ailleurs en France à l'époque. De plus, dans un pays de colonisation beaucoup de normes habituelles disparaissent par la force des choses. L'étude des gens d'Eglise est intéressante; signalons à ce sujet que l'origine géographique des prêtres a de l'importance en ce qui concerne la tenue des registres d'état civil, car on observait alors de grandes variations entre le nord et le midi de la France dans la rédaction des registres paroissiaux. Fort heureusement pour le Canada, ses premiers prêtres provenaient en majorité des régions situées au nord de la Loire et ils étaient en outre relativement plus lettrés que la moyenne des prêtres français de l'époque. C'est pourquoi nos registres du XVII siècle sont mieux tenus en moyenne que les registres français.

Au fil des six chapitres, l'auteur a l'habitude assez inusitée, notons-le, d'utiliser l'article devant les nombres relatifs (les $10,8 \%$ au lieu de 10,8\%): s'il s'agit d'un procédé littéraire, il va en contradiction avec l'ensemble des écrits de langue française en matière de démographie historique ou même d'histoire sociale.

A travers ce livre, remarquable à plus d'un titre, deux problèmes principaux surgissent constamment, sur lesquels nous insisterons quelque peu. Le premier se rapporte à l'observation statistique, c'est-à-dire à la collecte des données et à la critique des sources; il concerne donc l'appendice. Le second est également en partie d'ordre statistique et porte sur la question des réponses inconnues ou indéterminées, donc sur la distribution des "non déclaré" dans les divers tableaux de l'ouvrage.

\section{Critique des sources}

Si la présentation des sources manuscrites et bibliographiques se révèle excellente au début du livre, les explications sur la méthode employée paraissent malheureusement trop brèves. 
A lire M. Trudel, on a l'impression que l'opération de recensement n'a guère soulevé de difficultés et que nul n'a échappé aux investigations. Il est pourtant facile d'imaginer le nombre considérable de problèmes soulevés par semblable entreprise. Quel a été, dans cet effort, le rôle joué par chacune des sources ? Quelle part ont tenu en particulier les dictionnaires de Tanguay et de Godbout ? Comment ont été tranchés les cas controversés ? Quelle source l'emportait alors ? Surtout, quelle est la valeur respective de chaque source?

Certes, on ne peut exiger de l'auteur un exposé systématique et détaillé des problèmes rencontrés. Mais on aurait souhaité qu'il nous livre ses conclusions sur la valeur de son recensement. Or la tâche ne nous est guère facilitée à cet égard. Pas un mot n'est soufflé sur cet aspect capital de tout travail scientifique que représente la critique des sources. En somme, un nouvel outil est déposé entre les mains du lecteur sans que celui-ci en connaisse pleinement la valeur. Sans doute est-il probable qu'il s'agit de résultats extrêmement valables. Mais la question n'est pas là. Marcel Trudel ne nous explique pas suffisamment quel degré de confiance il accorde à ses sources et à son recensement. Lui seul est pourtant en mesure de nous renseigner à ce sujet.

La critique des sources constitue une opération délicate mais indispensable. Sans elle, l'incertitude subsiste. Si la reconstitution effectuée par M. Trudel doit être définitive, rien ne s'opposant à ce qu'elle le soit, il faut en connaître les marges d'erreur. Peut-être conviendrait-il de publier le dossier complet de la période 1627-1663, fiche par fiche.

Pour être plus concret, posons un problème simple mais précis: celui des enfants sans acte de décès. Nous choisissons nos exemples dans le dictionnaire Godbout, aux lettres A et B ${ }^{4}$.

Ignace Aubuchon est mentionné comme présent au recensement du 30 juin 1663, fils de Jacques et âgé de 5 ans, 9 mois (p. 267). Or Godbout le déclare décédé avant le recensement de 1666. L'acte de décès ne semble pas exister et n'a probablement jamais été fait. Comment la présence de cet enfant a-t-elle été établie ? A-t-on trouvé preuve de son décès après le 30 juin 1663 ou a-t-on seulement présumé qu'il était mort après cette date ? Si on en juge par les intervalles entre les naissances de ses frères et sœurs, cet enfant a pu mourir avant d'atteindre un an, soit vers 1658. Mais qu'en est-il en réalité ? La même question pourrait

4 Archange Godbout, "Nos ancêtres au XVIIe siècle", Rapport de l'Archiviste de la province de Québec (1951 à 1965), passim. 
être posée dans le cas de nombreux enfants. Citons quelques exemples:

Jacques Baucher, fils de Guillaume (p. 166)

Jacques Bertaut, fils de Jacques (p. 269)

Anne Biron, fille de Pierre (p. 169)

Mathurin Blondeau, fils de François (p. 170)

...etc.

Bien qu'on ne lui connaisse pas d'acte de décès non plus, Guillaume Bélanger, fils de François, est mentionné comme décédé avant le 30 juin 1663 (p. 167). Est-ce parce que Godbout l'affirme ou parce qu'on a trouvé une preuve ? La présence de tous ces enfants ne devrait-elle pas être considérée comme incertaine ?

\section{Répartition des "non déclaré"}

La question de la répartition des réponses inconnues peut se révéler très difficile en démographie historique, surtout quand ces cas indéterminés représentent une proportion non négligeable. Les "non déclaré" se distribuent-ils comme les "déclaré"? Ce peut être le cas mais pas nécessairement. Quand il s'agit de nombres relativement faibles, la répartition au prorata doit être faite, à moins que l'on ait des raisons de procéder autrement. Lorsque les renseignements inconnus ont de l'importance, il convient de rechercher prudemment la meilleure solution.

A la page 10 de son ouvrage, Marcel Trudel donne les proportions de réponses obtenues aux huit questions essentielles de ses dossiers individuels. Le défaut de renseignement est négligeable pour le nom, le prénom et le sexe; il n'est pas trop important pour l'âge, l'état matrimonial et l'origine mais s'accentue nettement dans le cas des signatures et des professions. Le lecteur est déjà mis en garde, mais ce n'est pas suffisant. Certes, l'auteur rappelle constamment, tout au long de son livre, l'existence des indéterminés. Mais comme il ne procède à aucune répartition, le résultat est identique à une répartition au prorata. Cette solution reste valable lorsque les "non déclaré" se répartissent à peu près comme les "déclaré". Est-ce toujours le cas ici ? En ce qui concerne l'origine, rien ne permet de penser que les cas inconnus diffèrent vraiment des cas connus. Il en va autrement pour l'âge, l'état matrimonial et surtout la profession et la faculté de signer. Dans ces derniers cas, il y a probablement un lien entre la nature de l'information et le fait qu'on soit informé ou non. Il se révèle alors impossible d'ignorer la rubrique 
"indéterminé" sans fausser les résultats. Une bonne évaluation doit être préférée à un nombre qui n'est exact qu'en apparence. Et le chercheur est seul à pouvoir suggérer la correction la plus adéquate.

Examinons de près l'exemple des nubiles aptes ou inaptes à signer (page 151). La tableau ci-dessous donne les éléments en cause:

\begin{tabular}{|c|c|c|}
\hline Réponse & Hommes & Femmes \\
\hline$\overline{\text { Connue }}$ & 887 & 357 \\
\hline — dont aptes à signer & 527 & 165 \\
\hline Inconnue & 406 & 278 \\
\hline Ensemble & 1293 & 635 \\
\hline
\end{tabular}

Se basant uniquement sur les cas connus, l'auteur conclut que 55,7\% des Canadiens de 1663 savaient signer leur nom. En ignorant les inconnus, il signifie nécessairement que la fraction d'analphabètes n'est pas plus élevée parmi les "non déclaré" qu'au sein des "déclaré". Il y a pourtant tout lieu de croire que l'on est beaucoup mieux informé sur les individus instruits que sur les illettrés. Plus un individu sait signer, plus il risque d'apparaître dans les archives avec sa signature. L'inverse est aussi vrai.

Voyons à quels résultats on arrive en tenant compte des réponses inconnues. Trois solutions sont possibles:

a) les "non déclaré" se répartissent en réalité comme les autres et la fraction des nubiles aptes à signer s'élève à $55,0 \%$, soit à une valeur à peu près analogue à celle de l'auteur;

b) les "non déclaré" savent tous signer et la proportion de lettrés passe alors à $71,4 \%$;

c) les "non déclaré" ne savent aucunement signer et l'analphabétisme n'exclut plus que 35,9\% des individus.

Comme il y a lieu de penser que la réalité est beaucoup plus proche de l'hypothèse " $c$ " que de l'hypothèse " $b$ ", on en déduit qu'à peine plus de $40 \%$ des Canadiens de l'époque savaient signer leur nom et que près de $60 \%$ étaient illettrés (au lieu de $45 \%$ ).

L'exemple des professions mériterait également un examen attentif. Il y a tout lieu de croire qu'on est mieux renseigné sur les individus qualifiés que sur les simples manouvriers. La proportion de $2 \%$ de domestiques paraît à cet égard extraordinairement faible. 
En guise de conclusion, souhaitons que les ordinateurs puissent s'emparer un jour de cette banque de données élaborée consciencieusement par M. Trudel, mettant ainsi à la portée de tous une véritable mine de renseignements.

Programme de recherche en

HUBERT CHARBONNEAU

démographie historique

Département de démographie

Université de Montréal 